Article

\title{
The Impact of Antenna Height on 3D-MIMO Channel: A Ray Launching Based Analysis
}

\author{
Qi Hong ${ }^{1, *}$, Jiliang Zhang ${ }^{2}$, Hui Zheng ${ }^{1}$, Hao Li ${ }^{1}$, Haonan Hu ${ }^{1}$, Baoling Zhang ${ }^{1}$, Zhihua Lai ${ }^{3}$ \\ and Jie Zhang 1,3 \\ 1 Department of Electronic and Electrical Engineering, University of Sheffield, Sheffield S10 2TN, UK; \\ hzheng@sheffield.ac.uk (H.Z.); hli33@sheffield.ac.uk (H.L.); haonan.hu@sheffield.ac.uk (H.H.); \\ bzhang13@sheffield.ac.uk (B.Z.); jie.zhang@sheffield.ac.uk (J.Z.) \\ 2 Shenzhen Graduate School, Harbin Institute of Technology, Harbin 518055, China; \\ zhangjiliang@hitsz.edu.cn \\ 3 Ranplan Wireless Network Design Ltd., Cambridge CB23 6DP, UK; zhihua.lai@ranplanwireless.com \\ * Correspondence: qhong2@sheffield.ac.uk; Tel.: +77-7447-900-312
}

\begin{abstract}
Three dimension (3D) Multi-input-multi-output (MIMO) scheme, which exploits another dimension of the spatial resource, is one of the enabling technologies for the next generation mobile communication. As the elevation angle in 3D-MIMO channel model might varies against the height of the base station transmit antenna, it has to be taken into account carefully. In this paper, the impact of antenna height on the channel characteristics of 3D MIMO channel is investigated by using the intelligent ray launching algorithm (IRLA). Three typical street scenarios, i.e., the straight street, the fork road and the cross road, are selected as benchmarks. On the basis of simulations, joint and marginal probability density functions (PDFs) of both the elevation angle of departure $(\mathrm{EAoD})$ and the elevation angle of arrival (EAoA) are obtained. The elevation angle spread (AS) and the delay spread (DS) under various antenna heights are also discussed. Simulation results indicate that the PDFs of EAoD and EAoA vary characteristics under different street scenarios. Moreover, the minimum value of the DS can be achieved when the antenna height is half of the building height.
\end{abstract}

Keywords: 3D MIMO; elevation angle; antenna height; elevation angle of departure; elevation angle of arrival; angle spread; delay spread

\section{Introduction}

One common feature of the research works on future wireless communication technologies is the pursuit of high spectral efficiency while multiple user equipments (UEs) are accessed into a base station (BS). Multi-User (MU) MIMO technologies are expected to play a key role to achieve high spectral efficiency [1]. To in-depth investigate MIMO, it is essential to have a good understanding of radio-propagation characteristics of transmission path between the BS and the mobile station (MS) [2]. Therefore, several models are proposed for the description of the radio channel [3-5]. In [3] and [4], the authors analyzed the channel characteristics by assuming the scatters are located uniformly within a circle or an ellipse. In [5], the author argued that stochastic geometry and random graph theory are indispensable tools for the analysis of wireless networks. For the conventional study on channel model, linear antenna arrays were assumed at horizontal direction [6-8]. The assumption is valid since the distance between the MS and the BS is much larger than the distance between the height of the BS antenna and the scatters around the MS [9]. However, with the UEs distributed in $3 \mathrm{D}$, the elevation angle should also be considered in channel modeling as it significantly influences the performance of MU-MIMO [10].

The first work on EAoD and EAoA was executed by T, Aulin in 1979 [11]. Then, more works have been researched to precisely describe the elevation angle. In 1990, a measurement in Tokyo based on $900 \mathrm{MHz}$ observed that the distribution of elevation angle is extremely closed to Gaussian distribution [12]. After that, Petrus derived the PDF of the multipaths' EAoD at the BS by assuming 
that the scattering objects were located uniformly within a circle around the MS [13]. In [14], M. Shafi extended the Spatial Channel Model (SCM) to 3D model by taking into account the elevation angle at the MS. Nawaz generalized a 3D scattering model for the macrocell environment with an MS located at the center of a 3D scattering semispheroid and a base station employing a directional antenna which located outside of the semispheroid in [15]. Many other works focusing on the elevation angle in different propagation environments are listed in [16-18]. Although previous works have well analyzed the distribution of EAoD and EAoA, the impact of antenna height on the channel characteristics is rarely considered.

With Internet of Things (IoT) and small cells are universally utilized, the antenna will be deployed at different heights. In order to optimize the antenna height to achieve better channel performance, we need to understand the impact of antenna height on parameters of wireless propagation, especially the EAoD and the EAoA. As introduced in [19], the heights of the antennas at both the BS and the MS, particularly the relative height between the BS and the building significantly influence the distribution of $\mathrm{EAoD}$ and $\mathrm{EAoA}$. The author also indicated that the AS will vary with the relative antenna height. In this paper, we mainly analyzed the characteristics of channel model under three different scenarios based on IRLA. The closed-form expressions are derived for the PDFs of EAoD and EAoA in terms of the antenna height. The effects of antenna height on the AS and the DS are also discussed.

This paper is organized as follows. Section 2 briefly introduces the IRLA and the experiment scenarios. In section 3, we derive the PDFs of EAoD and EAoA under various scenarios. Meanwhile, the AS and DS are also analyzed. The simulation results are presented in section 4. Finally, conclusions are given in section 5 .

\section{Methods and simulation scenarios}

\subsection{Intelligent ray launching algorithm (IRLA)}

Radio wave propagation prediction modeling is critical in wireless network planning and optimization [20]. Kinds of propagation models have been presented in decades which can be classified as empirical models and deterministic models [21-24]. The calculation of the empirical models channel characteristics is based on empirical factors such as carrier frequency and distance between the BS and the MS. These models are time-efficient but with lower accuracy. On the other hand, deterministic models consider the environmental information precisely such as the position of the buildings and the corresponding reflection factor. Thus they are time consuming but with higher accuracy.

The IRLA, which is utilized in our research, can be categorized as deterministic approaches. The concept of the IRLA is emitting the rays from the BS, and computing all the possible reflections and diffractions. Once the rays undergo reflections or diffractions, the additional loss will be added to the corresponding signal strength [25]. Inevitably, a small constant angle will be created to avoid distant area be covered when rays continue to diverge [26]. Therefore, a sphere structure is usually used to capture the missing rays.

In [27], Zhihua have originally presented the IRLA model. He indicated the details of the algorithm and justified the correctness in wireless network planning. In [28], the authors implemented the angular dispersion of ray launching to improve the accuracy. Then the IRLA model was validated by the measurement campaign [29].

\subsection{Experiment setup}

The simulation scenario is part of Paris center which is shown in Figure 1. The size of the area is $500 \mathrm{~m} \times 300 \mathrm{~m}$, and the average building height is approximately $21 \mathrm{~m}$ [30]. We configure the transmit power of the BS as $0 \mathrm{dBm}$ and the carrier frequency as $2.4 \mathrm{GHz}$. The antenna type is regarded as omnidirectional. The maximum reflection and diffraction number are 3 and 7 respectively. The parameters are list in Table 1. 
Table 1. Parameters setting of experiment scenario

\begin{tabular}{ccccccc}
\hline Size of area & $\begin{array}{c}\text { Average } \\
\text { building } \\
\text { height }\end{array}$ & $\begin{array}{c}\text { BS } \\
\text { power }\end{array}$ & $\begin{array}{c}\text { Carrier } \\
\text { frequency }\end{array}$ & Antenna type & $\begin{array}{c}\text { Max } \\
\text { reflection } \\
\text { number }\end{array}$ & $\begin{array}{c}\text { Max } \\
\text { diffraction } \\
\text { number }\end{array}$ \\
\hline $500 \mathrm{~m} \times 300 \mathrm{~m}$ & $21 \mathrm{~m}$ & $0 \mathrm{dBm}$ & $2.4 \mathrm{GHz}$ & Omnidirectional & 3 & 7 \\
\hline
\end{tabular}

Figure 1 illustrates that there are three typical street scenarios: straight street (red rectangular area), fork road (black rectangular area) and cross road (rose rectangular area). These three typical scenarios are displayed in Figure 2 and the parameters for each scenario are list in Table 2. During the simulation for each scenario, we deployed the position of the BS to be $(0,0, h)$, and the receivers to be at $\left(\mathrm{x}_{\mathrm{n}}, \mathrm{yn}_{\mathrm{n}} \mathrm{z}\right)$, both in the global coordinate system. The parameter $h$ is the height of the BS, and $n$ is the index of users arranging from 1 to 100000 which means 100000 receivers are uniformly located around the BS. We regard the angles collected above the antenna height as negative degree $\left(<0^{\circ}\right)$, and that below the antenna height as positive degree $\left(>0^{0}\right)$. Here we have not included the direct component.

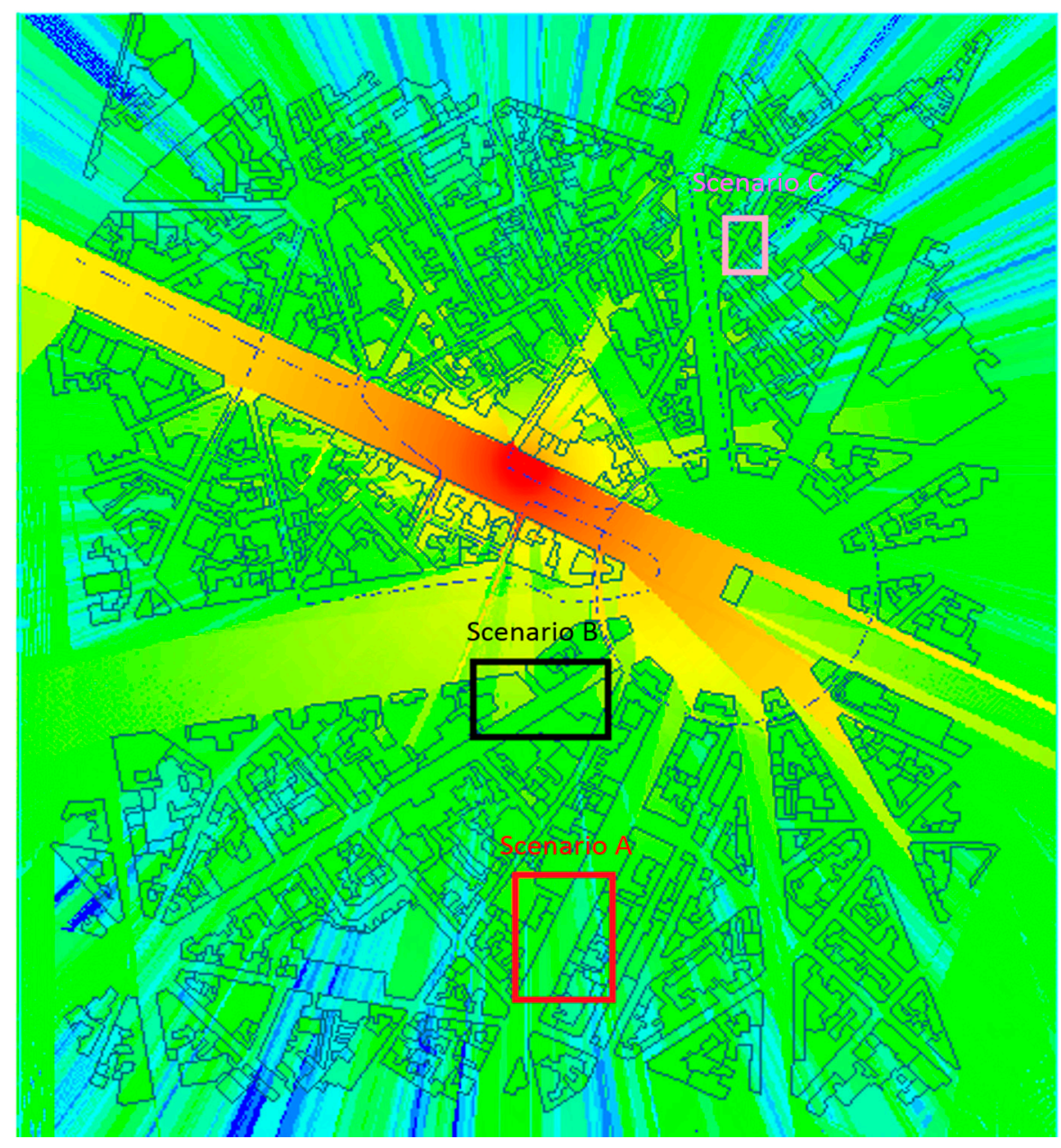

Figure 1. Part of Paris center (the red rectangular area, the black rectangular area and the rose rectangular area represent one of the straight streets, fork roads and cross roads respectively) [30]. 


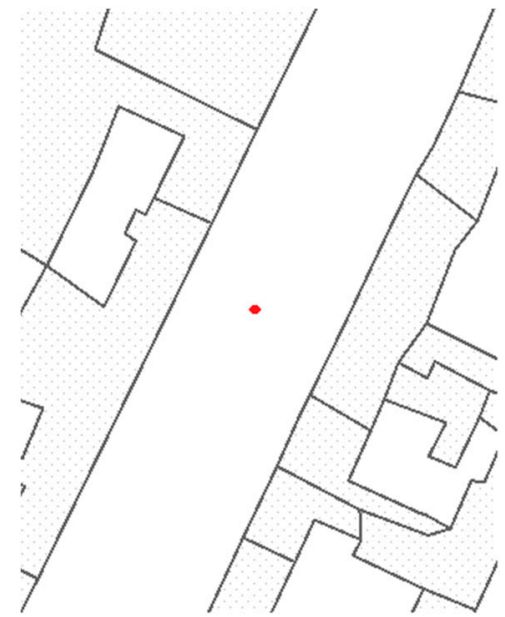

(a)

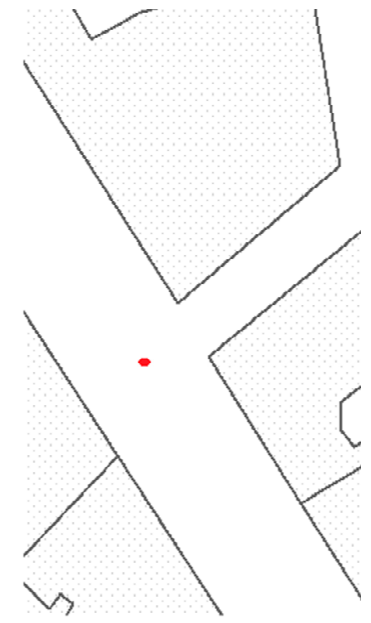

(b)

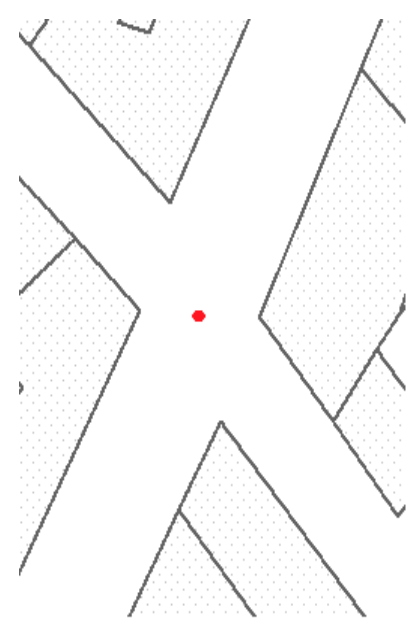

(c)

Figure 2. Three typical scenarios, (a) Description of the straight street; (b) Description of the fork road; (c) Description of the cross road.

Table 2. Parameters of three scenarios

\begin{tabular}{rrrrrr}
\hline & $\begin{array}{r}\text { Average } \\
\text { building height }\end{array}$ & $\begin{array}{r}\text { Length of } \\
\text { wider street }\end{array}$ & $\begin{array}{r}\text { Width of } \\
\text { wider street }\end{array}$ & $\begin{array}{r}\text { Length of } \\
\text { lower street }\end{array}$ & $\begin{array}{r}\text { Width of } \\
\text { lower street }\end{array}$ \\
\hline $\begin{array}{r}\text { Straight } \\
\text { street }\end{array}$ & $20 \mathrm{~m}$ & $80 \mathrm{~m}$ & $20 \mathrm{~m}$ & 0 & 0 \\
Fork road & $25 \mathrm{~m}$ & $80 \mathrm{~m}$ & $20 \mathrm{~m}$ & $40 \mathrm{~m}$ & $12 \mathrm{~m}$ \\
Cross road & $22 \mathrm{~m}$ & $40 \mathrm{~m}$ & $20 \mathrm{~m}$ & $40 \mathrm{~m}$ & $12 \mathrm{~m}$ \\
\hline
\end{tabular}

\section{Results}

\subsection{Scenario A: Straight street in Paris center}

Firstly, we studied the PDFs of EAOD and EAOA under the straight street in Figure 2(a). According to reflection and diffraction, each receiver can obtain multi rays with different path losses, EAoDs and EAoAs. Based on the IRLA, path losses, EAoDs and EAoAs of multi rays can be calculated. Using radian to express the elevation angle, and evenly divided the $(-\pi, \pi)$ into 101 intervals. Then the PDFs of EAoD and EAoA can be calculated after adding the weighting value (path loss). One simulation result (antenna height is $8 \mathrm{~m}$ ) is indicated below.

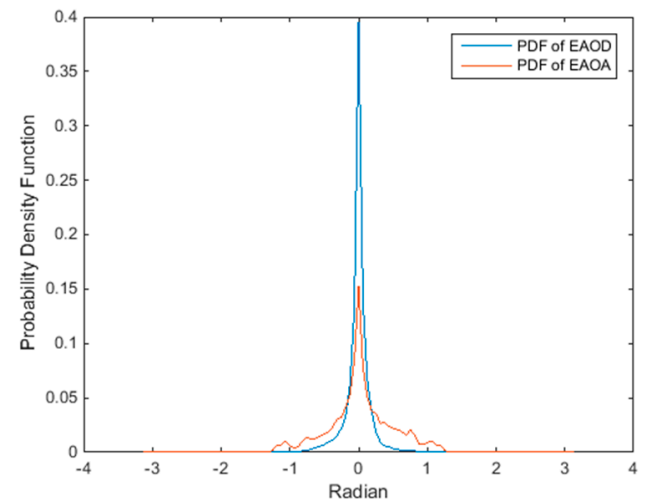

Figure 3. PDFs of EAoD and EAoA under straight street scenario when the antenna height is $8 \mathrm{~m}$.

As can be seen in Figure 3 that the PDF values of these two curves around 0 radian are obviously greater than the values away from 0 radian, and the sub-curves at the negative and positive sides of 
the peak are asymmetrical which have been verified by K. Kalliola in [18]. The author observed that a double-sided exponential function is more suitable for the PDF of elevation angles [18] which can be represented as:

$$
\begin{aligned}
& f_{\text {EAOD }}(x)=\left\{\begin{array}{ll}
\lambda_{1 E A O D} e^{-\lambda_{1 E A O D} x}, & x<0 \\
\lambda_{2 E A O D} e^{-\lambda_{2 E A O D} x}, & x>0
\end{array},\right. \\
& f_{E A O A}(x)=\left\{\begin{array}{l}
\lambda_{1 E A O A} e^{-\lambda_{1 E A O A} x}, \quad x<0 \\
\lambda_{2 E A O A} e^{-\lambda_{2 E A O A} x}, \quad x>0
\end{array},\right.
\end{aligned}
$$

Logarithm fitting the PDFs of EAoD and EAoA, we can finally obtain the value of $\lambda_{1 \mathrm{IEAD}}^{\prime} \lambda_{2 \mathrm{2EAD}}$, $\lambda_{1 \mathrm{EAOA}}$ and $\lambda_{2 \mathrm{2EAOA}}$, which are the logarithm of $\lambda_{1 \mathrm{EAOD}}, \lambda_{2 \mathrm{EAOD}}, \lambda_{1 \mathrm{EAOA}}$ and $\lambda_{2 \mathrm{EAOA}}$ (for example: $\lambda_{1 \mathrm{EAOD}}^{\prime}=$ $\left.-10 \times \log _{10} e \times \lambda_{1 E A O D}\right)$. For the simulation results of Figure $3, \lambda_{1 E A O D}^{\prime}=26.83, \lambda_{2 E A o D}^{\prime}=-36.48, \lambda_{1 E A o A}^{\prime}=$ $16.43, \lambda_{2 \mathrm{EAOA}}^{\prime}=-15.69$. The logarithm exponent value $\lambda^{\prime}$ of the exponential distribution will be different with various antenna heights which are listed in Figure 4.

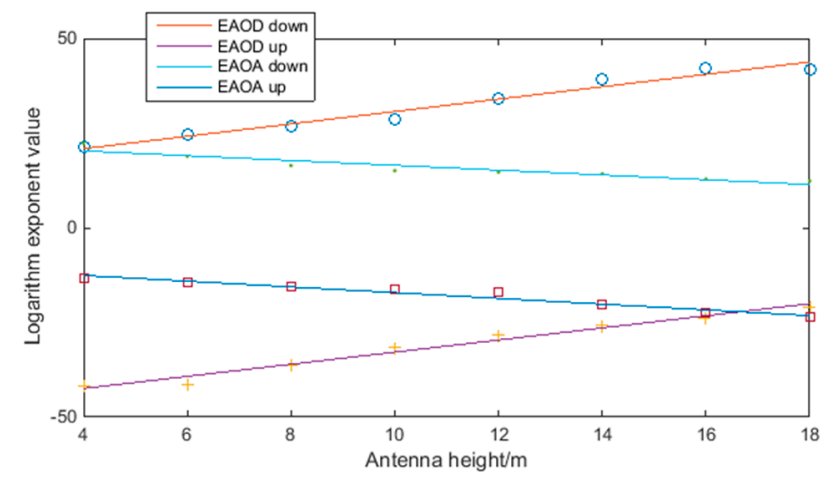

Figure 4. The statistic results of logarithm exponent values under straight street scenario with various antenna heights ( $4 \mathrm{~m}, 6 \mathrm{~m}, 8 \mathrm{~m}, 10 \mathrm{~m}, 12 \mathrm{~m}, 14 \mathrm{~m}, 16 \mathrm{~m}$ and $18 \mathrm{~m})$, the EAoD down, EAoD up, EAoA down and EAoA up represent $\lambda_{1 \mathrm{EAoD}}^{\prime} \lambda_{2 \mathrm{EAoD}}^{\prime} \lambda_{1 \mathrm{EAoA}}^{\prime}$ and $\lambda_{2 \mathrm{EAoA}}^{\prime}$ respectively.

Figure 4 illustrates that the logarithm exponent value is almost linear correction with the antenna height. We can derive that: $\lambda_{1 \mathrm{EAoD}}^{\prime}=1.64 \times \mathrm{m}+14.35, \lambda_{2 \mathrm{EAoD}}^{\prime}=1.60 \times \mathrm{m}-48.83, \lambda_{1 \mathrm{EAOA}}^{\prime}=-0.63 \times \mathrm{m}+$ $22.84, \lambda_{2 \mathrm{EAoA}}^{\prime}=-0.76 \times \mathrm{m}-9.55$. Here, $m$ represents the antenna height.

Finally, the PDFs of EAoD and EAoA relate to antenna height can be represented as:

$$
\begin{aligned}
& f_{E A O D}(x)=\left\{\begin{array}{l}
\frac{-(1.64 m+14.35)}{10 \times \log _{10} e} \times e^{\frac{(1.64 m+14.35) x}{10 \times \log _{10} e}}, x<0 \\
\frac{-(1.60 m-48.83)}{10 \times \log _{10} e} \times e^{\frac{(1.60 m-48.83) x}{10 \times \log _{10} e}}, x>0
\end{array}\right. \\
& f_{E A O A}(x)=\left\{\begin{array}{l}
\frac{-(-0.63 m+22.84)}{10 \times \log _{10} e} \times e^{\frac{(-0.63 m+22.84) x}{10 \times \log _{10} e}}, x<0 \\
\frac{-(-0.76 m-9.55)}{10 \times \log _{10} e} \times e^{\frac{(-0.76 m-9.55) x}{10 \times \log _{10} e}}, x>0
\end{array}\right.
\end{aligned}
$$

Using the PDFs results above, we characterize the AS and the DS which are used to define the angle dispersion and time dispersion of multipath components. In [13], the AS and the DS can be calculated as:

$$
\begin{gathered}
\sigma_{A S}=\sqrt{\frac{\sum_{i=1}^{L-1} P_{i} \theta_{i}^{2}}{\sum_{i=1}^{L-1} P_{i}}-\left(\frac{\sum_{i=1}^{L-1} P_{i} \theta_{i}}{\sum_{i=1}^{L-1} P_{i}}\right)^{2}}, \\
\sigma_{D S}=\sqrt{\frac{\sum_{i=1}^{L-1} P_{i} \tau_{i}^{2}}{\sum_{i=1}^{L-1} P_{i}}-\left(\frac{\sum_{i=1}^{L-1} P_{i} \tau_{i}}{\sum_{i=1}^{L-1} P_{i}}\right)^{2}},
\end{gathered}
$$


where $P_{i}$ is the ray power, $\theta_{i}$ and $\tau_{i}$ are the angle and delay of $i$ th multi rays. The statistic results of the AS and the DS are represented in Figure 5 and Figure 6 respectively.

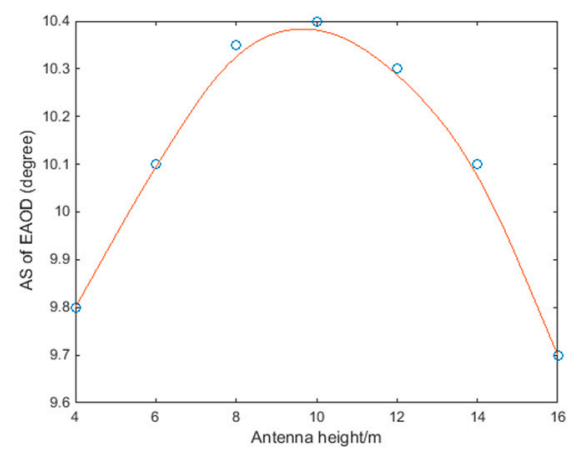

(a)

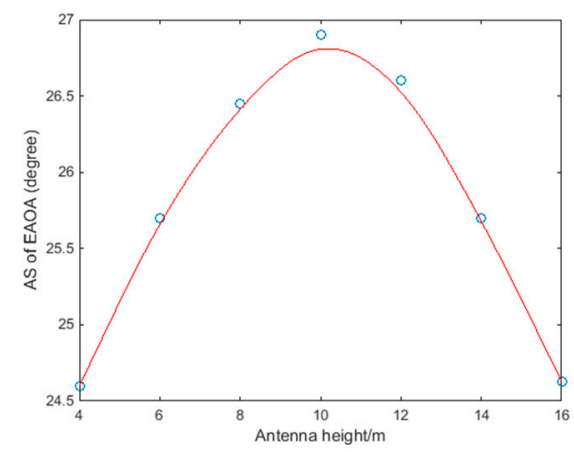

(b)

Figure 5. The AS under straight street with various antenna heights $(4 \mathrm{~m}, 6 \mathrm{~m}, 8 \mathrm{~m}, 10 \mathrm{~m}, 12 \mathrm{~m}, 14 \mathrm{~m}$ and $16 \mathrm{~m})$, (a) Description of the AS of EAoD; (b) Description of the AS of EAoA.

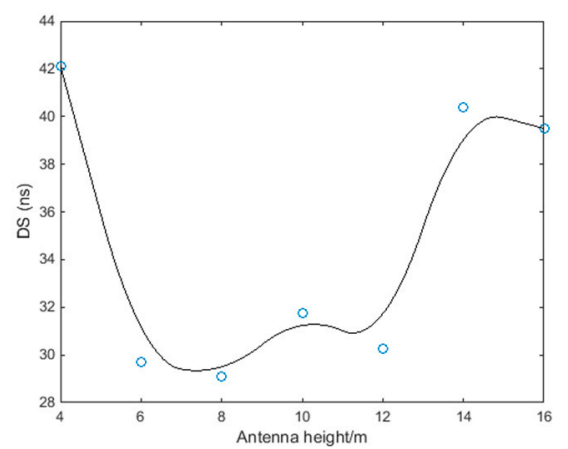

Figure 6. The DS with various antenna heights $(4 \mathrm{~m}, 6 \mathrm{~m}, 8 \mathrm{~m}, 10 \mathrm{~m}, 12 \mathrm{~m} 14 \mathrm{~m}$ and $16 \mathrm{~m})$.

In Figure 5, we can find that the value of the AS of EAoD is smaller than that of EAoA, and the variation range of the $\mathrm{AS}$ of $\mathrm{EAoD}$ is also smaller. The AS reachs its maximum value when the antenna height is half of the building height $(10 \mathrm{~m})$. Figure 6 exhibits that the value of the DS is nearly unchangable when the antenna height is around half of the building height and increases conspicuously when the antenna is near the bottom or the top of the buildings.

\subsection{Scenario B: Fork road in Paris center}

Then by using the same research steps in 3.1, we investigated the PDFs of EAoD and EAoA under fork road scenario as shown in Figure 2(b). The simulation result when the antenna height is $8 \mathrm{~m}$ is indicated in Figure 7.

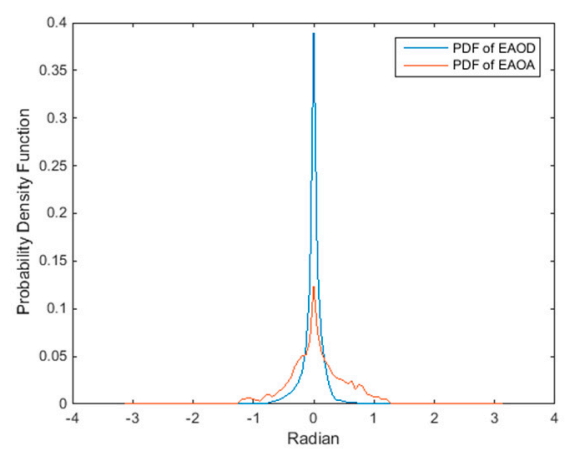

Figure 7. PDFs of EAoD and EAoA under fork road scenario when the antenna height is $8 \mathrm{~m}$. 
After fitting the curves under different antenna heights $(6 \mathrm{~m}, 8 \mathrm{~m}, 10,12 \mathrm{~m}, 14 \mathrm{~m}, 16 \mathrm{~m}, 18 \mathrm{~m}, 20 \mathrm{~m})$, the PDFs of EAoD and EAoA can be obtained as indicated in Figure 8.

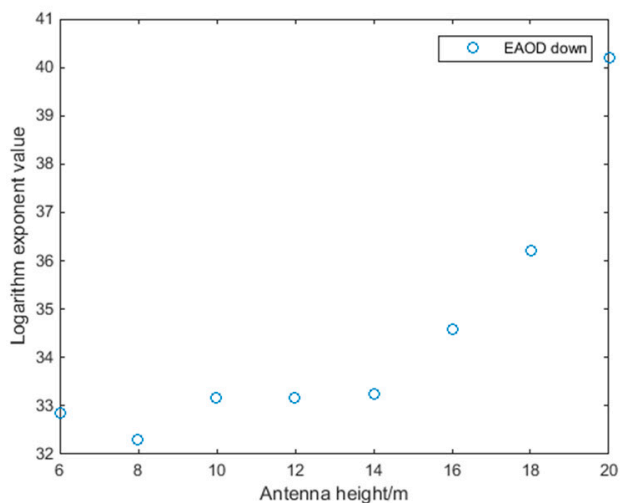

(a)

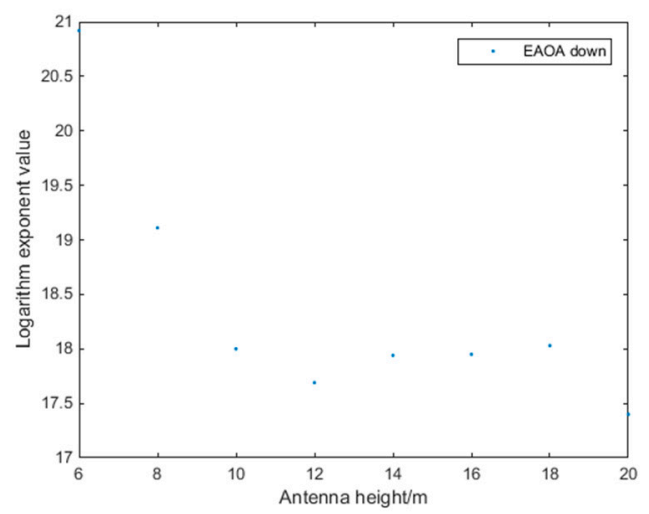

(c)

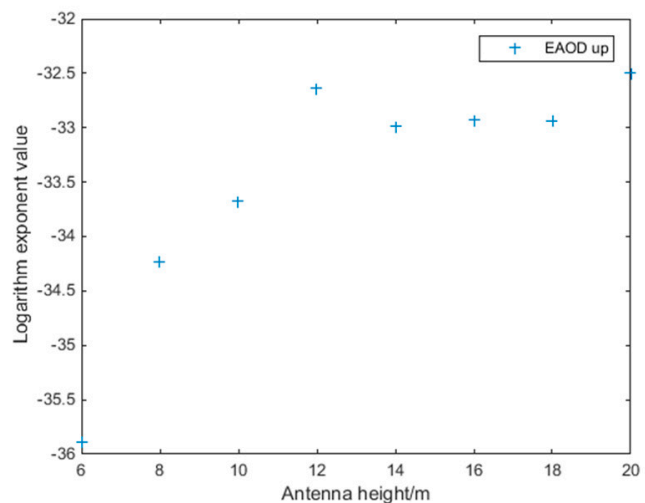

(b)

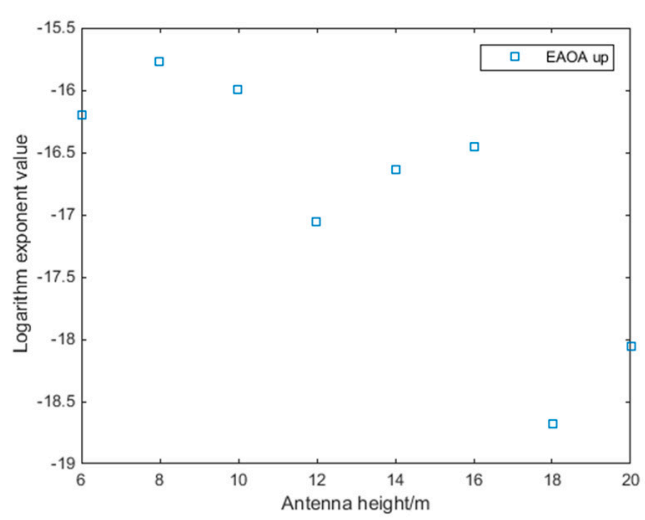

(d)

Figure 8. The statistic results of logarithm exponent values under fork road scenario with various antenna heights $(6 \mathrm{~m}, 8 \mathrm{~m}, 10 \mathrm{~m}, 12 \mathrm{~m}, 14 \mathrm{~m}, 16 \mathrm{~m}, 18 \mathrm{~m}$ and $20 \mathrm{~m})$. Here, the logarithm exponent values are the logarithm of $\lambda_{1 \mathrm{EAoD}}, \lambda_{2 \mathrm{EAoD}}, \lambda_{1 \mathrm{EAoA}}$ and $\lambda_{2 \mathrm{EAoA}}$ as shown in equations (1) and (2).

Finally, the PDFs of EAoD and EAoA related to antenna height can be calculated:

$$
\begin{aligned}
& f_{E A O D}(x)=\left\{\begin{array}{c}
\frac{-33.16}{10 \times \log _{10} e} \times e^{\frac{33.16 \times x}{10 \times \log _{10} e}}, x<0, m \leq 12.5 \\
\frac{-(0.61 m+25.57)}{10 \times \log _{10} e} \times e^{\frac{(0.61 m+25.57) x}{10 \times \log _{10} e}}, x<0,12.5<m<25 \\
\frac{-(0.3 m-36.59)}{10 \times \log _{10} e} \times e^{\frac{(0.3 m-36.59) x}{10 \times \log _{10} e}}, x>0, m \leq 12.5 \\
\frac{32.90}{10 \times \log _{10} e} \times e^{\frac{-32.90 \times x}{10 \times \log _{10} e}}, x>0,12.5<m<25
\end{array}\right. \\
& f_{\text {EAOA }}(x)=\left\{\begin{array}{c}
\frac{-(-0.26 m+21.17)}{10 \times \log _{10} e} \times e^{\frac{(-0.26 m+21.17) \times x}{10 \times \log _{10} e}}, x<0, m \leq 12.5 \\
\frac{-17.95}{10 \times \log _{10} e} \times e^{\frac{17.95 \times x}{10 \times \log _{10} e}, x<0,12.5<m<25} \\
\frac{16.5}{10 \times \log _{10} e} \times e^{\frac{-16.5 \times x}{10 \times \log _{10} e}}, x>0, m \leq 12.5 \\
\frac{-(-0.27 m-13.09)}{10 \times \log _{10} e} \times e^{\frac{(-0.27 m-13.09) \times x}{10 \times \log _{10} e}}, x>0,12.5<m<25
\end{array},\right.
\end{aligned}
$$


With the results obtained above, the AS and the DS of EAoD and EAoA can be gained as displayed in Figure 9 and Figure 10.

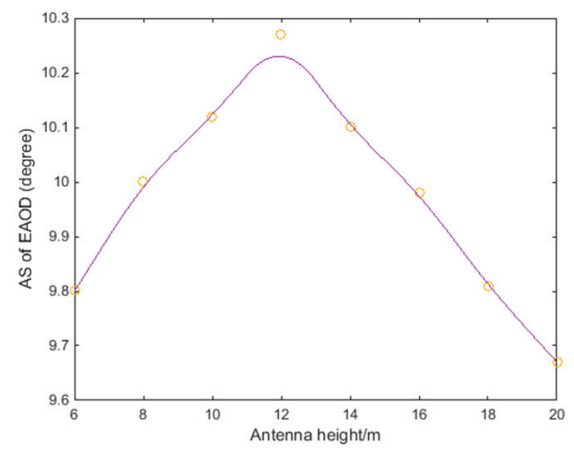

(a)

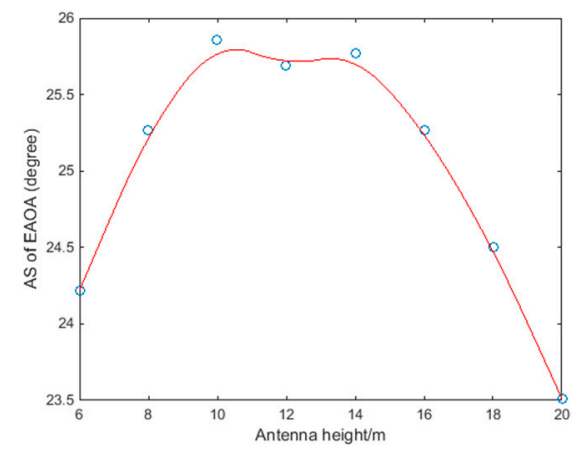

(b)

Figure 9. The AS under fork road scenario with various antenna heights $(6 \mathrm{~m}, 8 \mathrm{~m}, 10 \mathrm{~m}, 12 \mathrm{~m}, 14 \mathrm{~m}$, $16 \mathrm{~m}, 18 \mathrm{~m}$ and 20m), (a) Description of the AS of EAoD; (b) Description of the AS of EAoA.

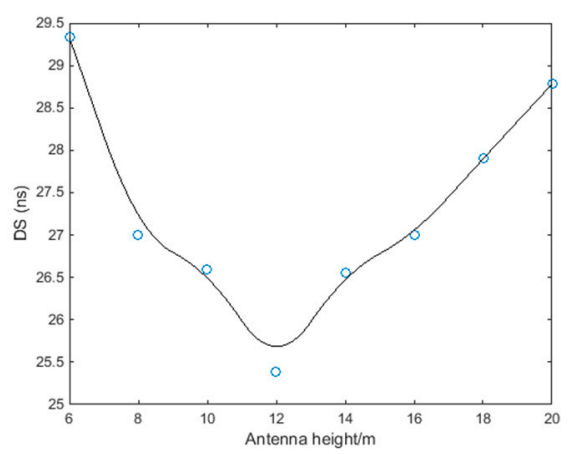

Figure 10. The DS under fork road scenario with various antenna heights $(6 \mathrm{~m}, 8 \mathrm{~m}, 10 \mathrm{~m}, 12 \mathrm{~m}, 14 \mathrm{~m}$, $16 \mathrm{~m}, 18 \mathrm{~m}$ and $20 \mathrm{~m}$ ).

It is obviously from Figure 9 that the ASs of EAoD and EAoA arrive the peak value when the antenna height is half of the building height $(10 \mathrm{~m})$. In Figure 10, we can conclude that minimum value of the DS can be achieved when the antenna height is half of the building height and the value arise when the antenna is near ground or top of the buildings.

\subsection{Scenario C: Cross road in Paris center}

Finally we researched the PDFs of EAoD and EAoA under cross road scenario as shown in Figure 2(c). The simulation result when the antenna height is $10 \mathrm{~m}$ is displayed in Figure 11.

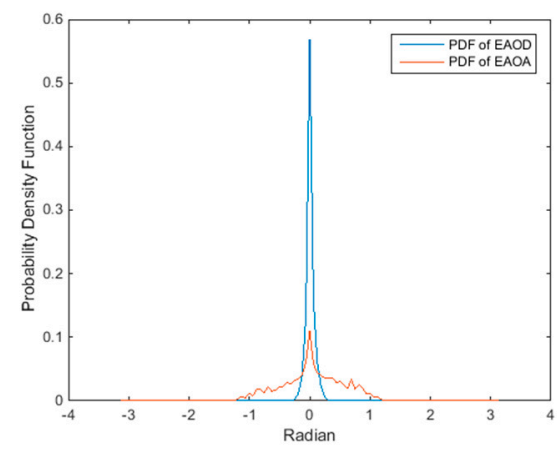

Figure 11. PDFs of EAoD and EAoA under cross road scenario when the antenna height is $10 \mathrm{~m}$. 
The logarithm exponent values of EAoD and EAoA can be obtained by fitting the curves through different antenna heights $(4 \mathrm{~m}, 6 \mathrm{~m}, 8 \mathrm{~m}, 10,12 \mathrm{~m}, 14 \mathrm{~m}, 16 \mathrm{~m}, 18 \mathrm{~m})$ which are exhibited in Figure 12.

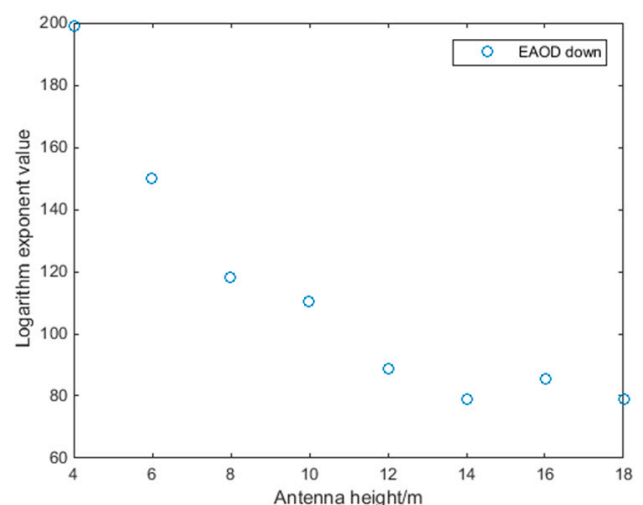

(a)

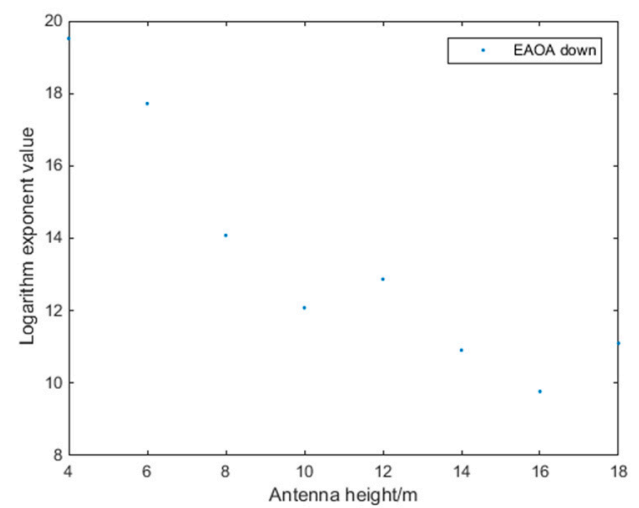

(c)

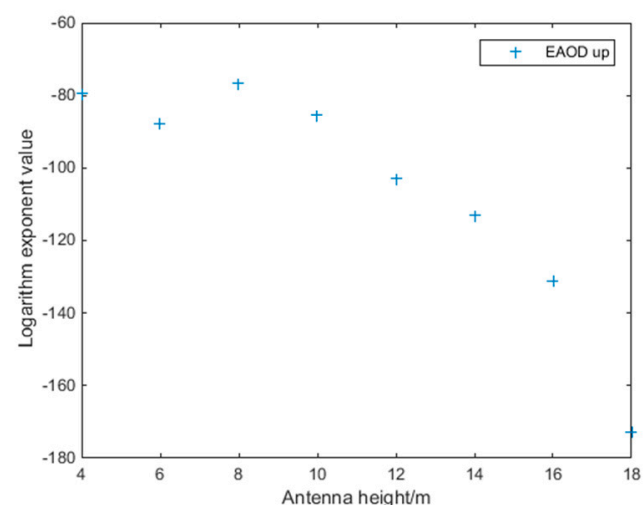

(b)

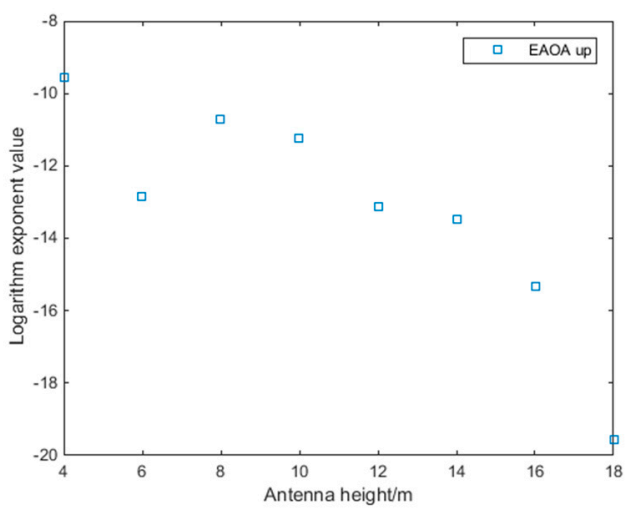

(d)

Figure 12. The statistic results of logarithm exponent values under cross road scenario with various antenna heights $(4 \mathrm{~m}, 6 \mathrm{~m}, 8 \mathrm{~m}, 10 \mathrm{~m}, 12 \mathrm{~m}$ and $14 \mathrm{~m}, 16 \mathrm{~m}, 18 \mathrm{~m})$. Here, the logarithm exponent values are the logarithm of $\lambda_{1 \mathrm{EAoD}}, \lambda_{2 \mathrm{EAoD}}, \lambda_{1 \mathrm{EAoA}}$ and $\lambda_{2 \mathrm{EAoA}}$ as shown in equations (1) and (2).

Finally, the PDFs of EAoD and EAoA can be represented as:

$$
\begin{aligned}
& f_{E A O D}(x)=\left\{\begin{array}{l}
\frac{-(-12.8 m+226.8)}{10 \times \log _{10} e} \times e^{\frac{(-12.8 m+226.8) x}{10 \times \log _{10} e}}, x<0, m \leq 11 \\
\frac{-86}{10 \times \log _{10} e} \times e^{\frac{86 \times x}{10 \times \log _{10} e}, x<0,11<m<22} \\
\frac{90}{10 \times \log _{10} e} \times e^{\frac{-90 \times x}{10 \times \log _{10} e}, x>0, m \leq 11} \\
\frac{-(-8.24 m+0.64)}{10 \times \log _{10} e} \times e^{\frac{(-8.24 m+0.64) x}{10 \times \log _{10} e}}, x>0,11<m<22
\end{array}\right. \\
& f_{E A O A}(x)=\left\{\begin{array}{c}
\frac{-(-0.73 m+22.36)}{10 \times \log _{10} e} \times e^{\frac{(-0.73 m+22.36) \times x}{10 \times \log _{10} e}}, x<0,0<m<22 \\
\frac{12}{10 \times \log _{10} e} \times e^{\frac{-12 \times x}{10 \times \log _{10} e}, x>0, m<11} \\
\frac{-(-0.66 m-4.6960)}{10 \times \log _{10} e} \times e^{\frac{(-0.66 m-4.6960) \times x}{10 \times \log _{10} e}}, x>0,11<m<22
\end{array},\right.
\end{aligned}
$$

The AS and the DS of EAoD and EAoA can be gained by the above results. 


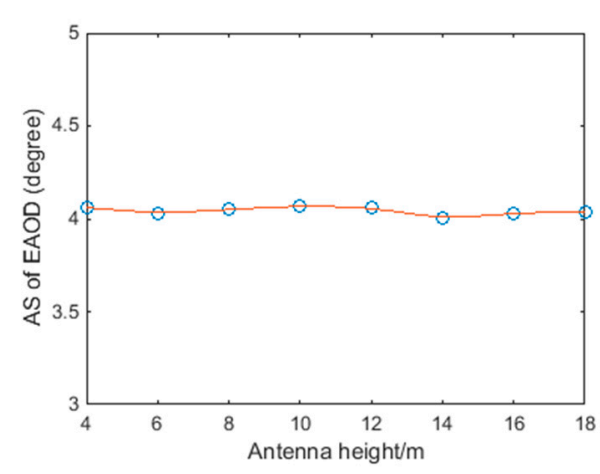

(a)

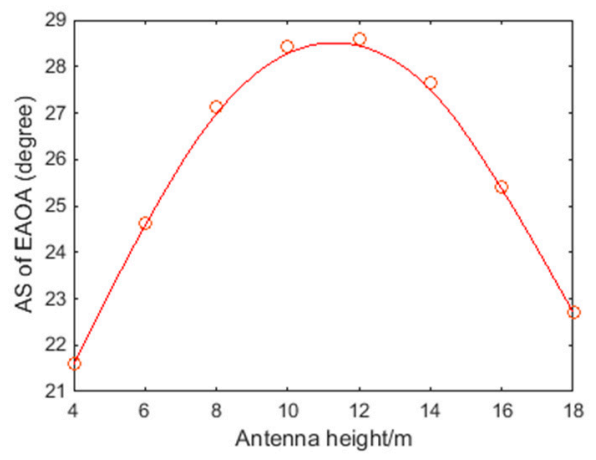

(b)

Figure 13. The AS under cross road scenario with various antenna heights $(4 \mathrm{~m}, 6 \mathrm{~m}, 8 \mathrm{~m}, 10 \mathrm{~m}, 12 \mathrm{~m}$ and $14 \mathrm{~m}, 16 \mathrm{~m}, 18 \mathrm{~m})$, (a) Description of the AS of EAoD; (b) Description of the AS of EAoA.

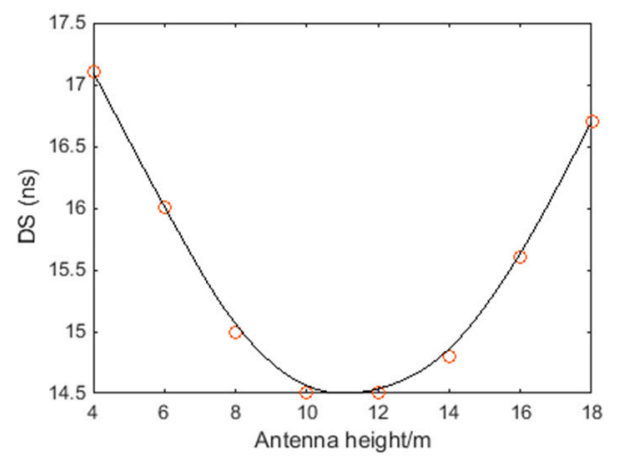

Figure 14. The DS under fork cross scenario with various antenna heights $(4 \mathrm{~m}, 6 \mathrm{~m}, 8 \mathrm{~m}, 10 \mathrm{~m}, 12 \mathrm{~m}$ and $14 \mathrm{~m}, 16 \mathrm{~m}, 18 \mathrm{~m})$.

Figure 13(a) presents that the AS of EAoD has little variation with different antenna heights. Figure 13(b) illustrates the AS of EAoA gains its maximum value when the antenna height is half of the building height $(11 \mathrm{~m})$ and drops gradually to the ground or the top of the buildings. The DS has the similar phonomenon with section 3.1 and 3.2 as shown in Figure 14.

\section{Discussion}

In this section we discuss the PDF characteristics of EAoD and EAoA under various road scenarios. Firstly we list the statistic logarithm exponent values of the PDF of EAoD and EAoA under the straight street scenario with various antenna heights $(4 \mathrm{~m}, 6 \mathrm{~m}, 8 \mathrm{~m}, 10 \mathrm{~m}, 12 \mathrm{~m}, 14 \mathrm{~m}, 16 \mathrm{~m}$ and $18 \mathrm{~m})$.

Table 3. Statistic logarithm exponent values of the PDF of EAoD and EAoA under straight street scenario with various antenna heights.

\begin{tabular}{lrrrrrrrr}
\hline & $\mathbf{4 m}$ & $\mathbf{6 m}$ & $\mathbf{8 m}$ & $\mathbf{1 0 m}$ & $\mathbf{1 2 m}$ & $\mathbf{1 4 m}$ & $\mathbf{1 6 m}$ & $\mathbf{1 8 m}$ \\
\hline EAoD $(x<0)$ & 21.26 & 24.51 & 26.83 & 28.7 & 34.18 & 39.32 & 42.14 & 41.78 \\
EAoD $(x>0)$ & -41.81 & -41.37 & -36.48 & -31.47 & -28.25 & -25.61 & -23.78 & -21.1 \\
EAoA $(x<0)$ & 22.29 & 18.9 & 16.43 & 15.09 & 14.7 & 14.25 & 12.85 & 12.37 \\
EAoA $(x>0)$ & -13.19 & -14.54 & -15.69 & -16.26 & -17.02 & -20.06 & -22.55 & -23.59 \\
\hline
\end{tabular}

Where $x$ is the randon variable of the exponential distribution shown in equations (1) and (2). $x<0$ represents the angle collected above the antenna height, and $x>0$ represents the angle collected below the antenna height. As we can see from Table 3, the logarithm exponent value $\lambda_{1 \mathrm{EAoD}}^{\prime}$ and $\lambda_{2 \mathrm{2} A \mathrm{AD}}$ (as 
shown in eqation (1)) linearly increase with the antenna height growing and the exponent value $\lambda_{1 \mathrm{EAOA}}^{\prime}$ and $\lambda^{\prime}{ }_{\mathrm{EEAOA}}$ (as shown in equation (2)) linearly decrease with the antenna height growing.

Then we list the PDF values under fork road with various antenna heights $(6 \mathrm{~m}, 8 \mathrm{~m}, 10 \mathrm{~m}, 12 \mathrm{~m}$, $14 \mathrm{~m}, 16 \mathrm{~m}, 18 \mathrm{~m}$ and $20 \mathrm{~m}$ ) in Table 4.

Table 4. Statistic logarithm exponent values of the PDF of EAoD and EAoA under fork road scenario with various antenna heights.

\begin{tabular}{lrrrrrrrc}
\hline & $\mathbf{6 m}$ & $\mathbf{8 m}$ & $\mathbf{1 0 m}$ & $\mathbf{1 2 m}$ & $\mathbf{1 4 m}$ & $\mathbf{1 6 m}$ & $\mathbf{1 8 m}$ & $\mathbf{2 0 m}$ \\
\hline $\mathrm{EAoD}(\mathrm{x}<0)$ & 32.86 & 32.31 & 33.16 & 33.16 & 33.25 & 34.58 & 36.2 & 40.19 \\
$\mathrm{EAoD}(\mathrm{x}>0)$ & -35.89 & -34.23 & -33.67 & -32.63 & -32.99 & -32.93 & -32.94 & -32.49 \\
$\mathrm{EAoA}(\mathrm{x}<0)$ & 20.92 & 19.11 & 18 & 17.69 & 17.94 & 17.95 & 18.03 & 17.4 \\
$\mathrm{EAoA}(\mathrm{x}>0)$ & -16.2 & -15.77 & -16 & -17.06 & -16.64 & -16.45 & -18.68 & -18.06 \\
\hline
\end{tabular}

From Table 4, two conclusions can be obtained:

- For the PDF of EAoD: when $x<0$, the logarithm exponent value $\lambda^{\prime}$ IEAoD is nearly unchanged when the antenna height is lower than half of the building height, but has a dramatically increase when the antenna height grows. However, when $x>0$, the logarithm exponent value $\lambda^{\prime}$ 2EAoD enhances when the antenna height is lower than half of the building height and then remains stable.

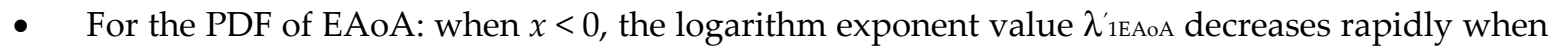
the antenna height is lower than half of the building height and then varies slightly. However, when $x>0$, the logarithm exponent value $\lambda_{2 \mathrm{EAOA}}^{\prime}$ is almost stable when the antenna height is lower than half of the building height, but has a significantly drop when the antenna height increases.

Finally, the PDF values under cross road scenario are listed in Table 5. The antenna heights vary from $4 \mathrm{~m}$ to $18 \mathrm{~m}$.

Table 5. Statistic logarithm exponent values of the PDF of EAoD and EAoA under cross road scenario with various antenna heights.

\begin{tabular}{lcccccccc}
\hline & $\mathbf{4 m}$ & $\mathbf{6 m}$ & $\mathbf{8 m}$ & $\mathbf{1 0 m}$ & $\mathbf{1 2 m}$ & $\mathbf{1 4 m}$ & $\mathbf{1 6 m}$ & $\mathbf{1 8 m}$ \\
\hline EAoD $(\mathrm{x}<0)$ & 199 & 149.8 & 118.3 & 110.5 & 88.88 & 79.08 & 85.5 & 79.05 \\
$\mathrm{EAoD}(\mathrm{x}>0)$ & -79.57 & -87.87 & -76.54 & -85.21 & -103 & -113.1 & -131.2 & -172.7 \\
$\mathrm{EAoA}(\mathrm{x}<0)$ & 19.52 & 17.72 & 14.08 & 12.08 & 12.87 & 10.91 & 9.77 & 11.1 \\
$\mathrm{EAoA}(\mathrm{x}>0)$ & -9.556 & --12.86 & -10.7 & -11.25 & -13.12 & -13.46 & -15.32 & -19.56 \\
\hline
\end{tabular}

From Table 5, we can conclude that:

- For the PDF of EAoD: when $x<0$, the logarithm exponent value reduces rapidly when the antenna height is lower than half of the building height, and gains the minimum value at $14 \mathrm{~m}$, then the value keeps the same. In contrast, when $x>0$ the logarithm exponent value is nearly unchangeable when the antenna height is lower than half of the building height, but has a dramatically decrease when the antenna height grows.

- For the PDF of EAoA: when $x<0$, the exponent value decreases with the antenna height arising. When $x>0$, the exponent value nearly unchanged when the antenna height is lower than half of the building height, but has a significantly drop when the antenna height increases.

Another phenomenon from the PDFs of EAoD and EAoA is that most numbers of EAoD and EAoA concentrate on 0 randian even though we ignore direct component as indicated in Figure 3, 7, 
11. Generally, the path distance of single reflection angle is smaller than the multi-path which makes the weighting value is higher. So we can conclude that the single reflection path plays a leading role for researching the distributions of EAoD and EAoA.

\section{Conclusions}

This article mainly researched the impact of antenna height on the channel characteristics under three typical road scenarios: straight street, fork road and cross road. At the beginning, we briefly introduced the IRLA and the simulation scenarios. Then, the closed-form expressions of the PDF of EAoD and EAoA were derived under various street scenarios, the related AS and DS were also presented. Finally, we discussed the PDF characteristics of EAoD and EAoA under various road scenarios.

The research in this paper is useful in 3D MIMO channel modeling. When considering the effect of the antenna height on the channel characteristic, the closed-form experssions of EAoD and EAoA derived under various road scanerios can be used. The value of tha AS and DS can also be applied to predict the channel property.

Acknowledgments: The research was supported in part by is3DMIMO, a research project supported by the European Commission.

Author Contributions: Qi Hong, Jiliang Zhang and Hui Zheng conceived and design the experiments, Jiliang Zhang, Hui Zheng and Hao Li performed the experiments; Qi Hong, Jiliang Zhang analyzed the data; Zhihua Lai and Haonan Hu contributed reagents/materials/analysis tools; Qi Hong, Baoling Zhang and Jie Zhang wrote the paper.

Conflicts of Interest: The authors declare no conflict of interest

\section{References}

1. L. P. David. Enhanced intercell interference coordination challenges in heterogeneous networks. IEEE Wireless Commun. 2011, Volume 18, pp.22-30.

2. G. D. Durgin; T. S. Rappaport. Theory of multipath shape factors for small-scale fading wireless channels. IEEE Trans. Antennas Propag. May. 2011, Volume 48, no. 5, pp.682-693.

3. R. B. Ertel; J. H. Reed. Angle and time of arrival statistics for circular and elliptical scattering models. IEEE J. Sel. Areas Commun. Nov. 1999, Volume 17, no. 11, pp.1829-1840.

4. N. M. Khan; et al. A generalized model for the spatial characteristics of the cellular mobile channel. IEEE Trans. Vehic. Tech. Sep. 2008, Volume 57, no. 1, pp.22-37.

5. M. Haenggi; et al. Stochastic geometry and random graphs for the analysis and design of wireless networks. IEEE J. Sel. Areas Commun. 2009, Volume 27, no. 7, pp.1029-1046.

6. R. B. Ertel; J. H. Reed. Angle and time of arrival statistics for circular and elliptical scattering models. IEEE J. Select. Areas Commun. Nov. 1999, Volume 17, pp.1829-1840.

7. W. C. Jakes; D. C. Cox; Microwave Mobile Communications, Wiley-IEEE Press, 1994.

8. R. Janaswamy. Angle and time of arrival statistics for the Gaussian scatter density model. IEEE Trans. Wireless Commun. July 2002, Volume 1, pp.488-497.

9. Mohammad; et al. Angle-of-Arrival statistics of a three-dimensional geometrical scattering channel model for indoor and outdoor propagation environments. IEEE Antenna and Wireless Letters. 2010, Volume 9, pp.946-949.

10. F. P. Fontan; et al. Statistical modelling of the LMS channel. IEEE Transsations on Vehcular Technology. 2001, Volume 50, pp.1549-1567.

11. T. Aulin. A modified model for the fading Signal at a mobile radio channel. IEEE Trans. Vehic. Tech. Aug. 1979, Volume 28, no. 3, pp.182-204. 
12. T. Taga. Analysis for mean effective gain of mobile antennas in land mobile radio environments. IEEE Trans. Vehic. Tech. May 1990, Volume 39, no. 2, pp.117-131.

13. P. Petrus; et al. Geometrical-based statistical macrocell channel model for mobile environments. IEEE Trans. Commun. 2002, Volume 50, no. 3, pp.495-502.

14. M. Shafi. The impact of elevation angle on MIMO capacity. IEEE ICC. June 2006, pp.4155-4160.

15. S. J. Nawaz. A generalized 3-D scattering model for a macrocell environment with a directional antenna at the BS. IEEE Trans. Vehic. Tech. Sep. 2010, Volume. 59, no. 7, pp.3193-3204.

16. J. D. Parsons; A. M. D. Turkmani. Characterizations of mobile radio signals: model description. IEE Proc. Dec. 1991, Volume 138, no. 6, pp.549-556.

17. S. Qu; T. Yeap. A Three-dimensional scattering model for fading channels in land mobile environment. IEEE Trans. Vehic. Tech. May 1999, Volume 48, no. 3, pp.765-781.

18. K. Kalliola; et al. Angular power distribution and mean effective gain of mobile antenna in different propagation environments. IEEE Trans. Vehic. Tech. Sept. 2002, Volume 51, no. 5, pp.823-838.

19. R. Janaswamy. Angle and time of arrival statistics for the Gaussian scatter density model. IEEE Trans. Wireless Commun. July 2002, Volume 1, no. 3, pp.488-497.

20. Y. Corre; Y. Lostanlen. Three-dimensional urban EM wave propagation model for radio network planning and optimization over large areas. IEEE Transactions on Vehicular Technology. 2009, vol. 58, no. 7, pp.3112-3123.

21. T. Kurner. Radio wave propagation part one "theoretical aspects". $1^{\text {st }}$ COST2100 Training School, Wroclaw, Poland, Feb. 2008.

22. Y. Lostanlen. Radio wave propagation part two "pratical aspects". $1^{\text {st }}$ COST2100 Training School, Wroclaw, Poland, Feb. 2008.

23. M. Klepal. Novel approach to indoor electromagnetic wave propagation modelling. Ph.D. dissertation, Czech Technical University, Prague. 2003.

24. J. Weng; X. Tu; Z. Lai; et al. Indoor Massive MIMO Channel Modelling Using Ray-Launching Simulation. International Journal of Antenna and Propagation. 2014.

25. R. Hoppe; G. Wolfle; F. Landstorfer. Accelerated ray optical propagation modelling for the planning of wireless communication networks. IEEE Raido and Wirelss Cofference, 1999, RAWCON 99.

26. B. Gschwendtner; G. Wolfle; B. Burl; F. Landstorfer. Ray tracing vs. ray launching in 3-d microcell modelling. European Personal and Mobile Communication Coference EPMCC95 10, 1995, pp.74-79.

27. Z. Lai; N. Bessis; G. De La Roche; et al. An intelligent ray launching for urban propagation prediction. The Third European Conference on Antennas and Propagation EuCAP. 2009, pp.2867-2871.

28. Z. LAI; N. Bessis; G. De La Roche; et al. A new approach to solve angular dispersion of discrete ray launching for urban scenarios. Loughborough Antennas \& Propagation Conference. 2009, pp.133-136.

29. Z. Lai; N. Bessis; G. De La ROche; et al. The characterisation of human-body influence on indoor 3.5 GHz path loss measurement. Second International Workshop on Planning and Optimization of Wireless Communication Networks. Sydney (Australia), 2010.

30. Outdoor LTE Small Cell Deployment on Lampposts: A Paris City Study. White paper, Available online: https://www.ranplan.co.uk/whitepaper/OutdoorSmallCellRanplan v4.pdf/,2014, 